\title{
The Impact of Three Waves of the COVID-19 Pandemic on the Characteristics of Primary Rhegmatogenous Retinal Detachments at a Tertiary Referral Centre
}

\section{Diana Dmuchowska (iD* \\ Izabela Cwalina* \\ Pawel Krasnicki \\ Joanna Konopinska (D) \\ Emil Saeed \\ Zofia Mariak \\ Iwona Obuchowska (DD}

Department of Ophthalmology, Medical University of Bialystok, Bialystok, 15-276, Poland

*These authors contributed equally to this work
Correspondence: Diana Dmuchowska Department of Ophthalmology, Medical University of Bialystok, 24a M. SklodowskiejCurie, Bialystok, 15-276, Poland

Tel +48857468372

Fax +48857468604

Email diana.dmuchowska@umb.edu.pl
Purpose: This study assessed the effect of three waves of the COVID-19 pandemic primarily on the number and timing of referrals due to rhegmatogenous retinal detachment (RRD) and secondarily on the demographic or clinical characteristics of patients.

Patients and Methods: A retrospective single-centre analysis of medical records included 247 eyes from 247 patients who underwent primary RRD repair with (phaco) vitrectomy between January 1, 2019, and May 31, 2021.

Results: The percentage of referrals due to primary RRD during the first year of pandemic (March 2020-February 2021) was 16.5\% ( $\mathrm{p}=0.179)$ lower than a year earlier. The percentage of referrals during the first, second and third wave of the pandemic was $48.4 \%, 18.5 \%$ and $26.1 \%$ ( $p=0.029,0.475,0.343)$ lower than in the corresponding months of 2019 . A rebound effect was observed only after the first wave. The numbers of referrals in April and May 2021 were similar as in the corresponding months of 2019. No significant differences were observed in the demographic and clinical characteristics of patients admitted before and during the COVID-19 pandemic, other than a tendency towards a higher representation of women, younger persons, longer duration of symptoms but better visual acuity in the latter group. Silicone oil tamponade was used more frequently during the pandemic than before. Conclusion: In this study, the COVID-19 pandemic affected the number and timing of referrals due to RRD and the clinical but not demographic characteristics of the patients. The effect lessened with the duration of the pandemic. Previously raised concerns regarding the delay in RRD referrals may no longer be valid during a potential fourth wave of the pandemic. The impact of the pandemic's waves should be analysed separately, as such an approach provides a better insight into the fluctuations in the number of referrals due to RRD than a year-to-year comparison.

Keywords: ophthalmic epidemiology, public health, SARS-CoV-2, lockdown

\section{Introduction}

COVID-19 pandemic affected healthcare in many aspects both from workers' and patients' perspectives. ${ }^{1-5}$ It also had a profound effect on ophthalmic referrals to emergency departments worldwide. ${ }^{6-10}$ One of the consequences of the pandemic was a decrease in the number of admissions for rhegmatogenous retinal detachment (RRD) surgeries. ${ }^{6,8,9,11-16}$ The degree to which the number of patients admitted for RRD surgeries has diminished across the world seems to be region-specific, with merely a $16 \%$ decrease observed in Australia, ${ }^{6}$ and over $60 \%$ and $42 \%$ reduction 
reported from the United Kingdom and Italy, respectively. ${ }^{9,16,17}$ The situation is even more complex because of regional differences in healthcare supply and demand and other local considerations.

The first case of COVID-19 in Poland was reported on March 4, 2020, with the first nationwide lockdown imposed on March 23. ${ }^{18,19}$ A total of three waves of the COVID-19 pandemic have been observed in Poland since then, with epidemic restrictions of various types being tightened and eased accordingly. The first nationwide lockdown was lifted in May 2020, but then the second one was imposed in October-December of the same year, followed by the third in March 2021, with maximum restrictions introduced in April 2021.

The Department of Ophthalmology at the Medical University of Bialystok (Poland) is the referral centre for the vast majority of patients with RRD from Podlaskie Voivodeship (a population of approximately 1.2 million). ${ }^{20}$ Surgical treatment for RRD is not offered by any private hospital in the region. Although the number of surgical procedures carried out at the Department of Ophthalmology has been reduced due to the COVID-19 pandemic, emergency services were available as usual.

In this study, we analysed whether the clinical features of primary RRD and the demographics of patients admitted with this condition differed depending on the pandemic stage. Most published reports of referrals due to RRD during the pandemic centred around the early impact of the first wave. ${ }^{6-9,11-14,17,21-23}$ To the best of our knowledge, this is the first study to analyse the yearto-year changes in the referrals due to RRD and to verify whether the number and characteristics of patients admitted with this condition changed across the three waves of the COVID-19 pandemic. We also verified whether the periodical lifting of epidemic restrictions was associated with a rebound effect on the referrals. In our opinion, the experiences presented in this report, the first one originating from a Central Eastern European referral centre, might help plan normalisation strategies in other cities and countries.

The aim of this study was to assess the effect of restrictions associated with the three waves of the COVID-19 pandemic primarily on the number and timing of referrals due to RRD and secondarily on the demographic and clinical characteristics of the patients. A timely referral is a critical factor in RRD management, as delayed treatment of this condition may lead to an irreversible loss of visual function, causing a substantial psychological and economic burden.

\section{Materials and Methods Study Design, Participants, Eligibility Criteria and Ethics}

The study was designed as a retrospective analysis of case sheets of consecutive case series treated at a single centre. The analysis included 247 eyes from 247 adults who underwent primary RRD repair at the Department of Ophthalmology, Medical University of Bialystok (Poland) between January 1, 2019, and May 31, 2021. The analysis included both cases that were referred from outpatient clinics, other, non-tertiary, centers and the patients who presented (referred) directly to our department. The only inclusion criterion was the diagnosis of primary RRD. Patients with tractional or serous retinal detachment $(\mathrm{RD})$ were not eligible for the analysis.

Information about the demographic (age, sex) and clinical data (incl. patient-reported duration of symptoms) was extracted from patients' casesheets. Visual symptoms were defined as the loss of vision, flashes, floaters and visual field defects. For statistical analysis, Snellen corrected distance visual acuity (CDVA) was converted into the logarithm of the minimum angle of resolution (logMAR). Anterior segment was assessed in the slit lamp. Axial length was measured with optical biometry and/or ultrasound. Macular involvement was assessed by fundoscopy, ultrasound and optical coherence tomography whenever possible. Macula-off RRD is defined as detachment involving the fovea.

The Polish government implemented and lifted epidemic restrictions according to the number of diagnosed COVID-19 cases, related morbidity and mortality, and capacity of the healthcare system. To analyse the year-toyear changes in the referrals due to RRD, the data for the first 12 months of the COVID-19 pandemic in Poland (from March 4, 2020, to February 28, 2021) were compared with the corresponding period a year earlier (from March 4, 2019, to February 29, 2020). Additionally, the results for the three waves of the COVID-19 pandemic in Poland were analysed using the dates of implementing and lifting epidemic restrictions as the cut-off points. ${ }^{24}$ The first wave was defined as a period between diagnosing the first COVID-19 case on March 4, 2020, and lifting most restrictions on May 31, 2020. The second wave was defined as a period between a sudden increase in the 
number of new COVID-19 cases on October 1, 2020, and lifting most related restrictions on December 31, 2020. The third was defined as a time from another sudden increase in the number of new COVID-19 cases on March 1, 2021, to a gradual loosening of related restrictions on April 30, 2021. The characteristics of referrals due to RRD during the three waves defined above were compared with the results for the corresponding months of 2019.

Starting on May 7, 2020, all patients treated at our centre underwent polymerase chain reaction (PCR) tests for COVID-19 before admission but after a clinical assessment. The recommendations in this matter had changed since April 1, 2021; patients no longer need to be tested on admission within three months after a positive result of the COVID-19 test or after being vaccinated. Four asymptomatic patients included in this analysis tested positively for COVID-19, two in November 2020 and another two in February 2021. One of those patients was operated on at our centre, whereas the other three were transferred to a hospital dedicated to patients with COVID-19 immediately after obtaining the positive result of the test. However, clinical characteristics of those three patients, other than the type of tamponade, were available and included in the present analysis.

The protocol of the study followed the provisions of the Declaration of Helsinki and was approved by the Local Bioethics Committee at the Medical University of Bialystok (decision no. APK.002.87.2021). All patients' data were anonymised upon collection. All study subjects gave written and informed consent for ophthalmological examination, treatment and the use of their clinical data for publication.

\section{Surgical Treatment}

All patients underwent pars plana vitrectomy with endolaser and either sulphur hexafluoride (SF6) or silicone oil tamponade. These were only medical indications (degree of PVR), and no others, that determined the kind of the tamponade. Phacovitrectomy was performed in all phakic cases. All surgical procedures, whether before or during the COVID-19 pandemic, were carried out by the same operators (PK and DAD) on the next day after admittance, with exclusion of weekends. The analysed group included three aphakic patients treated with simultaneous pars plana vitrectomy and secondary intraocular lens implantation.

\section{Statistical Analysis}

Statistical analyses were carried out with $\mathrm{R}$ software, version 4.0.5 (http://cran.r-project.org). Descriptive statistics included numbers (percentages) for nominal variables and means \pm standard deviations (SD) or medians and interquartile ranges $(\mathrm{Q} 1$, lower quartile $-\mathrm{Q} 3$, upper quartile) for continuous variables. Normal distribution of the analysed variables was verified with the Shapiro-Wilk test, on the basis of skewness and kurtosis values, as well as based on visual inspection of histograms. The results for pre-COVID and COVID populations were compared with the Pearson independence chi-square test, chi-square goodness-of-fit test or Fisher's exact test in the case of nominal variables and $t$-test or MannWhitney $U$-test in the case of continuous variables. Additionally, odds ratios (OR) or mean/median differences (MD) between pre-COVID and COVID populations were calculated as appropriate, along with their 95\% confidence intervals (CIs). Between-wave comparison of the three COVID waves was made with the Pearson independence chi-square test, chi-square goodness-of-fit test or Fisher's exact test in the case of nominal variables and ANOVA or Kruskal-Wallis test in the case of continuous variables.

Our primary outcome was the number of patients between non-COVID and COVID periods. A-priori sample size analysis revealed that assuming that we have $\alpha$ error of $5 \%$ and power of $80 \%$ for chi-square goodness-offit test with 1 degree of freedom (comparison of 2 periods) in total 88 patients were needed to achieve medium effect size $\mathrm{w}(0.3)$ and a total of 32 patients to achieve large effect size w (0.5). Sample size was calculated with GPower 3.1.9.2 software.

All tests were two-tailed, with $\alpha=0.05$.

\section{Results}

\section{Year-to-Year Comparison of the Impact of the COVID-19 Pandemic on the Number and Characteristics of Referrals Due to RRD}

Baseline characteristics of the patients are shown in Table 1 and S1. The majority of admitted patients, whether before or during the COVID-19 pandemic, were phakic, presented with macula-off RRD and underwent pars plana vitrectomy with silicone oil tamponade. 
Table I Characteristics of Patients with RRD Treated in the Pre-COVID Era and During the COVID-19 Pandemic

\begin{tabular}{|c|c|c|c|}
\hline & $\begin{array}{c}\text { Pre-COVID Year (March } 2019 \text { - } \\
\text { February 2020) }\end{array}$ & $\begin{array}{c}\text { First COVID Year (March } 2020 \text { - } \\
\text { February 202I) }\end{array}$ & $\mathbf{p}$ \\
\hline$N$ & 121 & 101 & 0.179 \\
\hline \multicolumn{4}{|l|}{ Sex, n (\%) } \\
\hline Female & $50(4 I .3)$ & $50(49.5)$ & 0.278 \\
\hline Male & 71 (58.7) & $5 \mathrm{I}(50.5)$ & \\
\hline Age, years, mean $\pm S D$ & $63.15 \pm 12.67$ & $60.53 \pm 12.77$ & 0.120 \\
\hline Duration of symptoms, days, median (QI;Q3) & $8.50(5.00 ; 26.25)$ & $10.00(7.00 ; 29.50)$ & 0.746 \\
\hline \multicolumn{4}{|l|}{ Operated eye, n (\%) } \\
\hline Left & 61 (50.4) & $46(45.5)$ & 0.557 \\
\hline Right & $60(49.6)$ & $55(54.5)$ & \\
\hline Pre-operative logMAR CDVA, median (QI;Q3) & $2.40(0.70 ; 2.70)$ & $1.70(1.00 ; 2.70)$ & 0.541 \\
\hline$A X L$, mean $\pm S D$ & $24.67 \pm 2.02$ & $24.62 \pm 1.88$ & 0.876 \\
\hline \multicolumn{4}{|l|}{ Lens status, $\mathrm{n}(\%)$} \\
\hline Phakic & $80(66.1)$ & $70(69.3)$ & 0.645 \\
\hline Pseudophakic & $39(32.2)$ & $28(27.7)$ & \\
\hline Aphakic & $2(1.7)$ & $3(3.0)$ & \\
\hline \multicolumn{4}{|l|}{ Macula status, n (\%) } \\
\hline On & $18(14.9)$ & 17 (16.8) & 0.831 \\
\hline Off & $103(85.1)$ & $84(83.2)$ & \\
\hline \multicolumn{4}{|l|}{ Tamponade, n (\%) } \\
\hline SF6 & 58 (47.9) & $40(39.6)$ & 0.268 \\
\hline Silicone oil & $63(52.1)$ & $61(60.4)$ & \\
\hline
\end{tabular}

Notes: Pre-COVID and COVID populations compared with $t$-test for age and AXL, Mann-Whitney U-test for duration of symptoms and logMAR of operated eye, chisquare goodness-of-fit test for number of patients, Pearson's independence chi-square test for sex, operated eye, tamponade and with Fisher exact test for lens status and macula.

Abbreviations: N, number; QI Q3, quartile I quartile 3; interquartile range; logMAR, the logarithm of the minimum angle of resolution; CDVA, corrected distance visual acuity; AXL, axial length; SF6, sulphur hexafluoride.

The percentage of referrals due to primary RRD during the first year of the pandemic was $16.5 \%$ lower than during the corresponding period a year earlier $(\mathrm{p}=0.179)$. No statistically significant differences were observed within the demographic and clinical characteristics of patients admitted during and before the COVID-19 pandemic. The following differences, though not statistically significant, were observed: higher representation of women ( 49.5 vs $41.3 \%, p=0.278$ ), younger persons $(60.53 \pm 12.77$ vs $63.15 \pm 12.67$ years, $\mathrm{p}=0.120)$, with a longer duration of symptoms $(10.00[7.00-29.5]$ vs $8.50[5.00-26.25]$ days, $\mathrm{p}=0.746)$ but better visual acuity (1.70 [1.00-2.70] vs 2.40[0.70-2.70] $\log$ MAR, $p=0.541)$ was observed in the population of patients admitted during the COVID-19 pandemic. No significant year-to-year differences were found in terms of axial length $(24.62 \pm 1.88$ vs $24.67 \pm 2.02 \mathrm{~mm}, \mathrm{p}=0.876)$, lens $(\mathrm{p}=0.645)$ and macula status ( 83.2 vs $85.1 \%$ macula off, $\mathrm{p}=0.831$ ). Silicone oil tamponade was used more frequently during the pandemic than before, but the difference was not significant (60.4 vs $52.1 \%, \mathrm{p}=0.268$ ).

\section{The Impact of Three Waves of COVID-I9 Pandemic on the Number and Characteristics of Referrals Due to RRD}

Baseline characteristics of the patients are presented in Table 2 and $\underline{S 1}$. The percentage of referrals due to primary RRD during the first, second and third wave of the pandemic was $48.4 \%, 18.5 \%$ and $26.1 \%(p=0.029, p=0.475$ and $\mathrm{p}=0.343$, respectively) lower than during the corresponding months of 2019 (Figure 1). The decrease in the number of referrals was the longest during the first wave; a sudden decline in the number of referrals was observed 


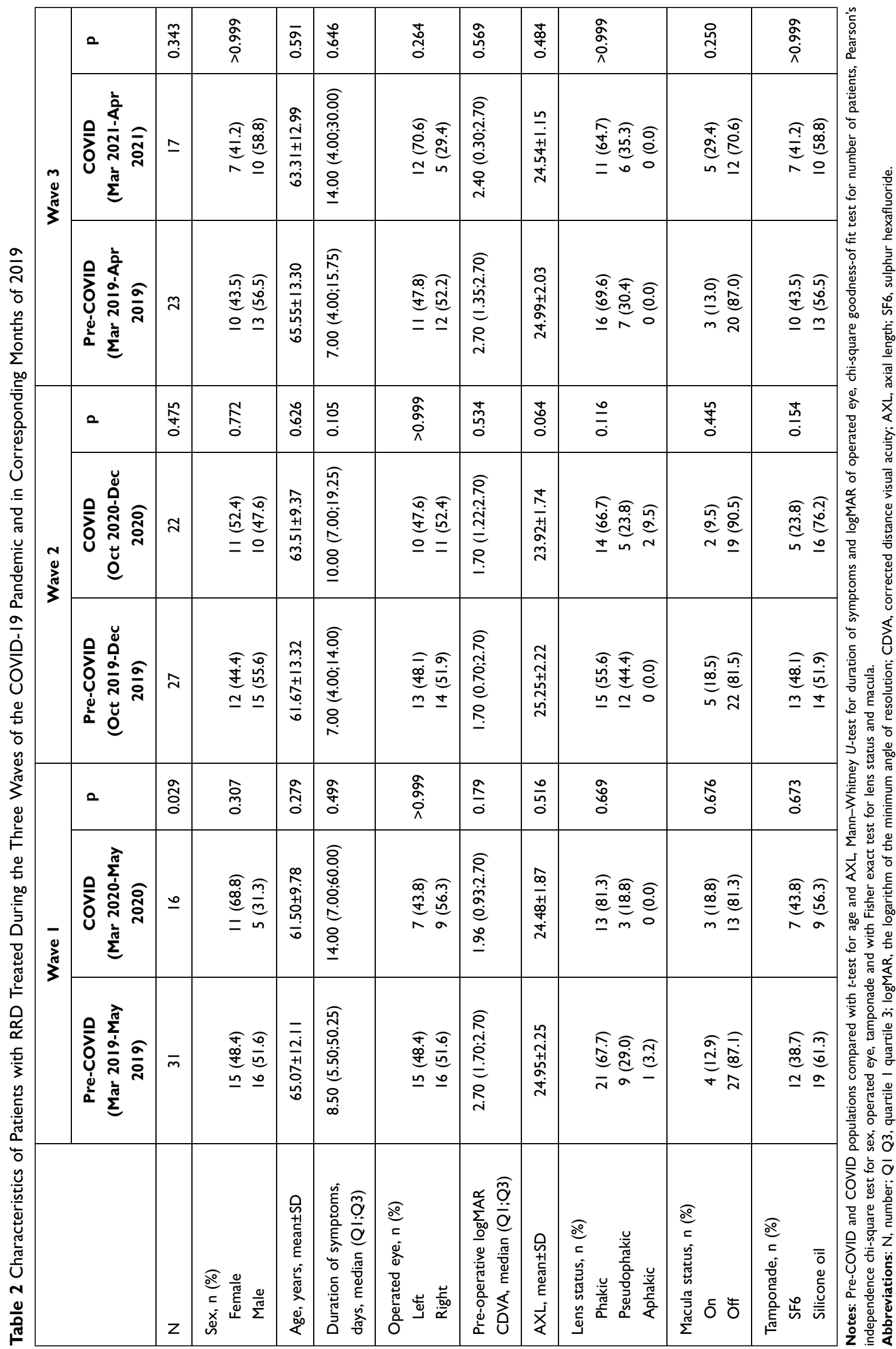




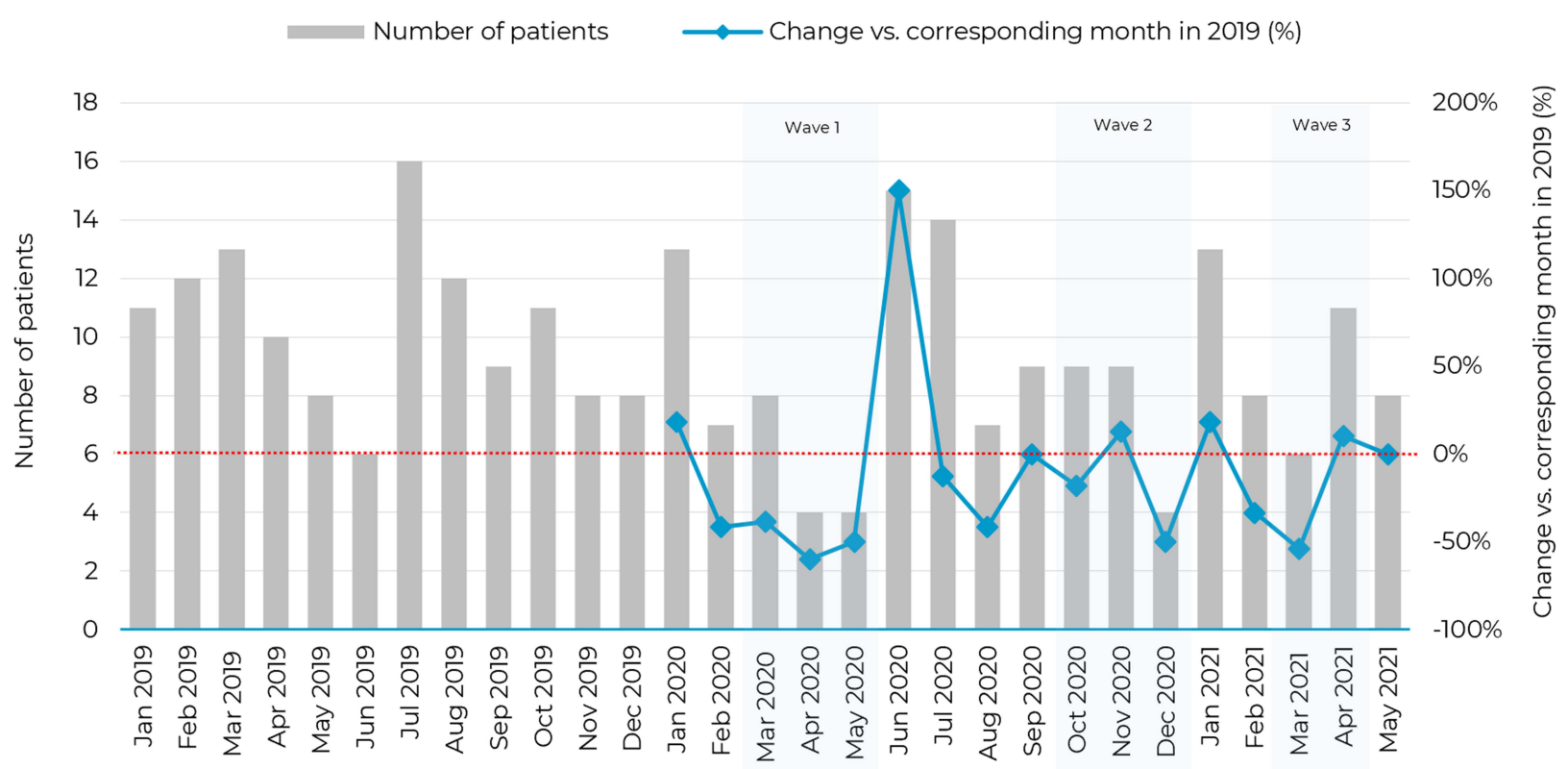

Figure I Monthly number of patients undergoing primary rhegmatogenous retinal detachment surgeries between January 2019 and May 202I (total $n=247$ ). Blue line represents \% change in number for a given month vs corresponding month in 2019 with reference on the right axis (eg - $18.2 \%$ for Oct 2020 means that number of operated patients was 18.2\% lower than in Oct 2019) (raw data in Table S2).

already in February 2020 and continued for four months (Table S2). The decrease during the second and third wave was less evident. A rebound effect was observed only after the first wave of the pandemic, in June 2020, when the percentage of referrals due to RRD increased by $150 \%$ as compared to June 2019. No rebound effect occurred after the second and third wave. The number of referrals after the first wave was essentially the same as in the corresponding months of 2019: 14 vs 16 in July, 7 vs 12 in August and 9 vs 9 in September. The same tendency was also observed after the second wave: 13 vs 13 in January and 8 vs 12 in February. During the third wave of the pandemic, in April 2021, the number of referrals due to RRD was no lower than in April 2019 (11 vs 10), and a similar tendency was also observed in May 2021 (8 vs 8 in May 2019).

No statistically significant differences were found when the demographic and clinical characteristics of patients admitted during the three waves of the pandemic were compared with the characteristics of patients treated during the corresponding months of 2019. While the differences were not statistically significant, patients admitted during the three waves of the pandemic tended to report a longer duration of symptoms but presented with better visual acuity than those treated during the corresponding months of 2019. No significant period-to-period differences were observed with regard to patients' sex and age, axial length, lens and macula status.

Furthermore, no significant differences in the number of referrals, demographic and clinical characteristics of RRDs were found in the between-wave comparison (Table 3).

\section{Discussion}

According to the literature, the COVID-19 pandemic contributed to a decrease (by $16 \%$ to over $60 \%$ ) in the number of RRD surgeries performed worldwide. ${ }^{6,8,9,11,13,14,17}$ While the percentage of referrals due to RRD at our centre was $16.5 \%(p=0.179)$ lower than the previous year, up to $48.4 \%(\mathrm{p}=0.029)$ decrease in the number of RRDs surgeries was observed during the first wave of the pandemic. This implies that the impact of the pandemic should be analysed within shorter periods rather than on a year-toyear basis. However, to the best of our knowledge, none of the previous studies centred around the impact of individual waves of the pandemic on the number of performed RRD surgeries.

According to the most plausible scenario, lockdowns associated with consecutive waves of the pandemic contributed to a decrease in the number of referrals due to $\mathrm{RRD}$, with subsequent rebound effect after easing the restrictions. ${ }^{11,14,16}$ Interestingly, at our centre, the rebound 
Table 3 Characteristics of Patients with Primary Rhegmatogenous Retinal Detachment Treated During the Three Waves of the COVID-19 Pandemic

\begin{tabular}{|c|c|c|c|c|}
\hline & $\begin{array}{c}\text { COVID Wave I } \\
\text { (Mar 2020-May 2020) }\end{array}$ & $\begin{array}{c}\text { COVID Wave } 2 \\
\text { (Oct 2020-Dec 2020) }\end{array}$ & $\begin{array}{c}\text { COVID Wave } 3 \\
\text { (Mar 202I-Apr 202I) }\end{array}$ & $\mathbf{p}$ \\
\hline N & 16 & 22 & 17 & 0.569 \\
\hline \multicolumn{5}{|l|}{ Sex, n (\%) } \\
\hline Female & II (68.8) & II (52.4) & $7(4 \mid .2)$ & 0.280 \\
\hline Male & $5(31.3)$ & $10(47.6)$ & $10(58.8)$ & \\
\hline Age, years, mean $\pm S D$ & $61.50 \pm 9.78$ & $63.51 \pm 9.37$ & $63.31 \pm 12.99$ & 0.688 \\
\hline Duration of symptoms, days, median (QI;Q3) & $14.00(7.00 ; 60.00)$ & $10.00(7.00 ; 19.25)$ & $14.00(4.00 ; 30.00)$ & 0.770 \\
\hline \multicolumn{5}{|l|}{ Operated eye, n (\%) } \\
\hline Left & $7(43.8)$ & $10(47.6)$ & $12(70.6)$ & 0.235 \\
\hline Right & $9(56.3)$ & II (52.4) & $5(29.4)$ & \\
\hline Pre-operative logMAR CDVA, median (QI;Q3) & $1.96(0.93 ; 2.70)$ & $1.70(1.22 ; 2.70)$ & $2.40(0.30 ; 2.70)$ & 0.896 \\
\hline $\mathrm{AXL}$, mean $\pm \mathrm{SD}$ & $24.48 \pm 1.87$ & $23.92 \pm 1.74$ & $24.54 \pm 1.15$ & 0.216 \\
\hline \multicolumn{5}{|l|}{ Lens status, n (\%) } \\
\hline Phakic & $13(8 \mid .3)$ & $14(66.7)$ & II (64.7) & 0.536 \\
\hline Pseudophakic & $13(18.3)$ & $5(23.8)$ & $6(35.3)$ & \\
\hline Aphakic & $0(0.0)$ & $2(9.5)$ & $0(0.0)$ & \\
\hline \multicolumn{5}{|l|}{ Macula status, n (\%) } \\
\hline On & $3(18.8)$ & $2(9.5)$ & $5(29.4)$ & 0.313 \\
\hline Off & $13(8 \mid .3)$ & $19(90.5)$ & $12(70.6)$ & \\
\hline \multicolumn{5}{|l|}{ Tamponade, n (\%) } \\
\hline SF6 & $7(43.8)$ & $5(23.8)$ & $7(4 \mid .2)$ & 0.373 \\
\hline Silicone oil & $9(56.3)$ & $16(76.2)$ & $10(58.8)$ & \\
\hline
\end{tabular}

Notes: COVID populations during three waves compared with ANOVA for age and AXL, Kruskal-Wallis test for duration of symptoms and logMAR of operated eye, chisquare goodness-of fit test for number of patients, Pearson's independence chi-square test for sex, operated eye, tamponade and with Fisher exact test for lens status and macula.

Abbreviations: N, number; QI Q3, quartile I quartile 3; logMAR, the logarithm of the minimum angle of resolution; CDVA, corrected distance visual acuity; AXL, axial length; SF6, sulphur hexafluoride.

effect, with an up to $150 \%$ increase in the percentage of referrals, was observed after the first wave of the pandemic but not after the second. This phenomenon might be associated with the fact that the decrease in referrals due to RRD was marked more evident during the first than during the second and third waves of the pandemic.

Notably, a discrepancy between the lockdown-related measures and COVID-19 morbidity and mortality has been observed in Poland. ${ }^{18,19}$ The toughest restrictions were imposed during the first wave when the number of newly diagnosed COVID-19 cases was relatively low. We hypothesised that the decrease in the number of patients presenting with RD was not necessarily associated with delayed referrals but rather with resignation from seeking medical advice. The aetiology of such patients' behaviour might be complex, involving both imposed epidemic restrictions and psychological factors. ${ }^{25}$ Furthermore, according to Napoli et al the lack of adequate and precise legal indications for surgeons and patients could result in excessive reduction in the volume of surgical interventions during a pandemic era. ${ }^{3-5}$

Among others, concern about COVID-19 infection acquired through ocular transmission, expressed not only in the scientific literature but also in the media, as well as inadequate availability of the personal protective equipment were the source of concern at the beginning of pandemic. $^{26}$ The level of fear of COVID-19 was shown to be modulated by demographic, social and psychological factors. $^{25,27,28}$ Plausibly, those factors might also influence patients' behaviour, contributing to postponing/abandoning hospital visit to mitigate the related risk of COVID-19 infection. Hence, the decrease in the number of referrals 
might be only partially explained by a hindered access to ophthalmologists, especially given that the services of outpatient clinics and elective procedures in Poland were suspended only during the first wave of the pandemic. Further, patients referring to our centre with the symptoms of RD are consulted at the emergency room both with or without a previous visit to an outpatient clinic. As our analysis included solely referrals due to primary RRD, and the cases of traumatic RD were excluded, it is unlikely that the decrease in the number of admissions reflected the impact of epidemic restrictions on trauma-predisposing activities, eg work, mobility, social gatherings and physical activity.

We assume that the level of fear and anxiety lessened with the introduction of the anti-COVID-19 vaccination program. Our analysis demonstrated a tendency toward an increased number of referrals to our centre, especially during the third wave. This increase might be related to a growing proportion of vaccinated persons and the resultant decrease in the level of COVID-19 fear. ${ }^{29}$ The proportion of partly or fully vaccinated persons in Poland has increased substantially, from $5.8 \%$ at the beginning of the third wave (March 1, 2021) to $22.6 \%$ at the end of April 2021 and $36.1 \%$ on May $31,2021 .^{30}$ Since May 9, 2021, all adults in Poland can register for the vaccination. Additionally, we speculate that the level of fear may have diminished thanks to available scientific data regarding low risk of COVID ocular transmission, ${ }^{26}$ availability of personal protective equipment and potential antiviral properties of drops used postoperatively. ${ }^{31}$

Delayed treatment of RRD may have long-term implications, including psychological and economic burden. The visual prognosis after RRD surgery depends in part on the duration of the disease and macular status. ${ }^{32,33}$ Median patient-reported duration of symptoms (vision loss, flashes, floaters or visual field defects) after the pandemic outbreak was longer than a year ago and increased considerably during all three waves. However, it needs to be stressed that visual disturbances are a subjective ailment, and as such, do not constitute a reliable marker of RRD duration and should be interpreted with caution.

Unlike the patient-reported duration of symptoms, the other two parameters analysed in this study, the proportions of patients with macula-off RRD and proliferative vitreoretinopathy, the latter reflected by the type of tamponade, are more reliable, objective markers of RRD duration. Surprisingly, the proportion of patients with macula-off RRD increased only during the second wave of the pandemic, and a slight decrease in this parameter was observed during a year-to-year comparison and when the results for the first and third waves of the pandemic were compared with the corresponding months of 2019. This observation stays in opposition to the results of previous studies in which the COVID-19 pandemic contributed to a various-degree increase in the prevalence of macula-off RRD in various countries. ${ }^{11,13,14,16,17,34}$ Importantly, however, those studies covered shorter periods. According to Kaupke et $\mathrm{al}^{22}$ the number of referrals due to macula-off RRD at a German ophthalmology department remained unchanged during the pandemic, which is consistent with our findings.

It needs to be stressed that the rate of macula-off RRD in our patients was relatively higher than in other studies. Even in the pre-COVID era, the proportion of referrals due to macula-off RRD at our centre was much higher than the prevalence of this condition estimated in large epidemiological studies (approximately $85 \%$ vs $50-60 \%$ ). ${ }^{35,36}$ Such a high proportion of patients admitted with macula-off $\mathrm{RRD}$, being a consequence of delayed referral, explains the frequent use of vitrectomy and silicone oil tamponade at our centre $(52 \%$ of RRD surgeries in the pre-COVID era and $60 \%$ during the COVID-19 pandemic).

A higher prevalence of proliferative vitreoretinopathy related to a longer duration of RRD during the pandemic has been reported previously. ${ }^{11,13,14,34}$ In our analysis, the more frequent occurrence of proliferative vitreoretinopathy was reflected by the higher rate of silicone oil tamponade. An increase in the proportion of RRD surgeries involving silicone oil tamponade was observed both on year-to-year comparison and during the second and third waves of the pandemic, implying a delay in the referrals due to RRD. Patients admitted during the first wave had better visual acuity, lower rates of macula-off RRD and less often required silicone oil tamponade. We assume that this group of patients who because of higher level of health awareness would refer to our centre regardless of the pandemic, whereas others resigned from the treatment or postponed it until the interwave period.

COVID-19 is a source of fear, stress and anxiety. The levels of those are modulated by a plethora of factors, among them sex and age. ${ }^{25,37}$ Hence, one may expect that patients admitted for RRD surgeries during the COVID-19 pandemic had different demographic characteristics than those treated in the pre-COVID era. However, similar to previous studies, we did not find 
significant differences in the age and sex distribution of patients admitted during the pandemic and a year earlier. $^{13,14,22,34}$

We assumed that pseudophakic patients, with a history of previous ophthalmic surgery, might be more likely to refer for RRD treatment. However, similar to Arjmand et $\mathrm{al}^{13}$ and Jasani et $\mathrm{al}^{34}$ we did not find such a relationship in our analysis.

This study has some unquestioned strengths. First, unlike in most previous studies, our analysis covered a relatively long period, and hence, included patients who postponed their visit because of lockdown and referred to the clinic once the epidemic restrictions have been eased. Second, aside from the year-to-year comparison, we analysed also the impact of the three waves of the COVID-19 pandemic on the number and characteristics of referrals due to RRD; such an approach might be crucial for planning patients' admission during a potential fourth wave.

We are well aware of the potential limitations of this study. Due to the relatively small sample size, some period-to-period differences might not reach the threshold of statistical significance. Further, it is unclear whether the results obtained under a specific epidemiological situation of Podlaskie Voivodeship could be generalised onto other populations affected by the COVID-19 pandemic.

Regarding the condition of the Polish healthcare during the COVID-19 pandemic, the availability of human resources and life-supporting machines were flexibly adjusted to the demand, and although the system was very close to reaching its maximum capacity, the latter has never been exceeded. This analysis covers a specific geographical area, patient population and healthcare system. It needs to be stressed that the situation of healthcare providers in Podlaskie Voivodeship was relatively good compared with other administrative regions of Poland. Also, the number of COVID-19 cases there was lower than in other regions.

\section{Conclusion}

COVID-19 pandemic affected the number and timing of referrals due to RRD and the clinical characteristics of the patients. This impact lessened with consecutive waves of the pandemic. Patients' demographics remained unchanged. Previously raised concerns regarding the pandemic-related delay in referrals due to RRD may no longer be valid.
The results of this single-centre analysis may reflect the future situation in countries that are currently most affected by the pandemic. Our findings do not support previously raised concerns about a substantial increase in the number of emergency referrals due to RRD and the severity of this condition once the COVD-19-related restrictions are lifted. ${ }^{9,17}$ We cautiously hypothesise that, in an equivalent setting, a potential fourth wave of the pandemic might not influence the number of referrals due to RRD and patients' characteristics. The results of this study might help plan normalisation strategies when the pandemic is over.

The impact of individual waves of the pandemic should be analysed separately, as such an approach provides a better insight into the fluctuations in the number of referrals due to RRD than a year-to-year comparison.

\section{Data Sharing Statement}

All the materials and information will be available upon an email request to the corresponding author. Names and exact data of the study participants may not be available owing to patient confidentiality and privacy policy.

\section{Ethics Approval}

The protocol of the study followed the provisions of the Declaration of Helsinki and was approved by the Local Bioethics Committee at the Medical University of Bialystok (decision no. APK.002.87.2021). All study subjects gave written and informed consent for ophthalmological examination, treatment and the use of their clinical data for publication.

\section{Consent for Publication}

The participants have consented to the submission of the results of the study to the journal.

\section{Author Contributions}

DD and IC worked on the conception, study design, execution, acquisition of data, analysis and interpretation and main text with figures and tables. PK, JK, ES, ZM and IO worked on the analysis and interpretation and on the main text.

All authors took part in drafting, revising or critically reviewing the article; gave final approval of the version to be published; have agreed on the journal to which the article has been submitted; and agreed to be accountable for all aspects of the work.

\section{Funding}

There is no funding to report. 


\section{Disclosure}

The authors have no commercial or proprietary interest in any of the products or companies mentioned in this article. The authors report no conflicts of interest in this work.

\section{References}

1. Bohlken J, Schömig F, Lemke MR, Pumberger M, Riedel-Heller SG. [COVID-19 pandemic: stress experience of healthcare workers a short current review]. Psychiatr Prax. 2020;47(4):190-197. doi:10.1055/a-1159-5551. [German]

2. Raymond E, Thieblemont C, Alran S, Faivre S. Impact of the COVID-19 outbreak on the management of patients with cancer. Target Oncol. 2020;15(3):249-259. doi:10.1007/s11523-020-00721-1

3. Nioi M, Napoli PE, Finco G, Demontis R, Fossarello M, d'Aloja E. Fear of the COVID-19 and medical liability. Insights from a series of 130 consecutives medico-legal claims evaluated in a single institution during SARS-CoV-2-related pandemic. Signa Vitae. 2021;17 (4):79-85.

4. Nioi M, Napoli PE, Lobina J, Fossarello M, d'Aloja E. COVID-19 and Italian healthcare workers from the initial sacrifice to the mRNA vaccine: pandemic chrono-history, epidemiological data, ethical dilemmas, and future challenges. Front Public Health. 2020;2020 (8):591900. doi:10.3389/fpubh.2020.591900

5. Napoli PE, Nioi M, d'Aloja E, Fossarello M. Safety recommendations and medical liability in ocular surgery during the COVID-19 pandemic: an unsolved dilemma. J Clin Med. 2020;9(5):1403. doi: $10.3390 / \mathrm{jcm} 9051403$

6. Kam AW, Gunasekaran N, Chaudhry SG, Vukasovic M, White AJR, Fung AT. Reduction in ophthalmic presentations to Australian emergency departments during the COVID-19 period: are we seeing the full picture? Clin Ophthalmol. 2021;15:341-346. doi:10.2147/OPTH. S289467

7. Toro MD, Brézin AP, Burdon M, et al. Early impact of COVID-19 outbreak on eye care: insights from EUROCOVCAT group. Eur J Ophthalmol. 2021;31(1):5-9. doi:10.1177/1120672120960339

8. Al-Khersan H, Kalavar MA, Tanenbaum R, et al. Emergent ophthalmic surgical care at a tertiary referral center during the COVID-19 pandemic. Am J Ophthalmol. 2021;222:368-372. doi:10.1016/j. ajo.2020.08.044

9. Wickham L, Hay G, Hamilton R, et al. The impact of COVID policies on acute ophthalmology services-experiences from Moorfields Eye Hospital NHS foundation trust. Eye. 2020;34 (7):1189-1192. doi:10.1038/s41433-020-0957-2

10. Ting DSJ, Deshmukh R, Said DG, Hs D. The impact of COVID-19 pandemic on ophthalmology services: are we ready for the aftermath? Ther Adv Ophthalmol. 2020;2020(12):2515841420964099. doi:10.1177/2515841420964099

11. Patel LG, Peck T, Starr MR, et al. Clinical presentation of rhegmatogenous retinal detachment during the COVID-19 pandemic: a historical cohort study. Ophthalmology. 2021;128(5):686-692. doi:10.1016/j.ophtha.2020.10.009

12. Akram H, Dowlut MS, Karia N, Chandra A. Emergency retinal detachment surgery during COVID-19 pandemic: a national survey and local review. Eye. 2020:1-2. doi:10.1038/s41433-020-01187-1

13. Arjmand P, Murtaza F, Eshtiaghi A, Popovic MM, Kertes PJ, Eng KT. Impact of the COVID-19 pandemic on characteristics of retinal detachments: the Canadian experience. Can J Ophthalmol. 2021;56(2):88-95. doi:10.1016/j.jcjo.2020.12.008

14. Awad M, Poostchi A, Orr G, Kumudhan D, Zaman A, Wilde C. Delayed presentation and increased prevalence of proliferative vitreoretinopathy for primary rhegmatogenous retinal detachments presenting during the COVID-19 pandemic lockdown. Eye. 2021;35 (4):1282-1283. doi:10.1038/s41433-020-1056-0
15. Poyser A, Deol SS, Osman L, et al. Impact of COVID-19 pandemic and lockdown on retinal detachments. Eye. 2020:35(8):2322-2323. doi:10.1038/s41433-020-01137-x

16. Franzolin E, Longo R, Casati S, Ceruti P, Marchini G. Influence of the COVID-19 pandemic on admissions for retinal detachment in a tertiary eye emergency department. Clin Ophthalmol. 2021;15:2127-2131. doi:10.2147/OPTH.S307407

17. Poyser A, Deol SS, Osman L, et al. Impact of COVID-19 pandemic and lockdown on eye emergencies. Eur $J$ Ophthalmol. 2020:1120672120974944. doi:10.1177/1120672120974944.

18. Regulations of the minister of health, regarding the announcement of a state of epidemic on the territory of the republic of Poland, polish journal of laws of 2020. Available from: https://isap.sejm.gov.pl/isap. nsf/DocDetails.xsp?id=WDU20200000491. Accessed July 19,2021.

19. World Health Organization. Available from: https://covid19.who.int/ region/euro/country/pl. Accessed July 19, 2021.

20. Brzostowska M, Grobicka E, Jelińska-Hrynkiewicz J, et al. Regions of Poland 2020. Zakład Wydawnictw Statystycznych; August 14, 2020. Available from: https://stat.gov.pl/download/gfx/portalinforma cyjny/en/defaultaktualnosci/3315/5/14/1/regions_of_poland_2020. pdf. Accessed July 19, 2021..

21. Dawkins RCH, Paul RA, Allen PJ, Yeoh J, Essex RW. Dramatic fall in retinal detachment presentations during the COVID-19 pandemic: collateral damage due to COVID-19. Asia Pac J Ophthalmol. 2021;10(1):121. doi:10.1097/APO.0000000000000339

22. Kaupke N, Spitzer MS, Kromer R. [Treatment of retinal detachment during the COVID-19 pandemic: did patients with retinal detachment seek treatment later during the COVID-19 pandemic? Results from a German university eye hospital]. Ophthalmologe. 2020. doi:10.1007/s00347-020-01248-6. [German]

23. Schranz M, Georgopoulos M, Sacu S, et al. Incidence and surgical care of retinal detachment during the first SARS-CoV-2 lockdown period at a tertiary referral center in Austria. PLoS One. 2021;16(3): e0248010. doi:10.1371/journal.pone.0248010

24. Dong E, Du H, Gardner L. An interactive web-based dashboard to track COVID-19 in real time. Lancet Infect Dis. 2020;20(5):533-534. doi:10.1016/S1473-3099(20)30120-1

25. Długosz P. Predictors of mental health after the first wave of the COVID-19 pandemic in Poland. Brain Sci. 2021;11(5):544. doi:10.3390/brainsci11050544

26. Napoli PE, Nioi M, d'Aloja E, Fossarello M. The ocular surface and the coronavirus disease 2019: does a dual "Ocular Route" exist? J Clin Med. 2020;9(5):1269. doi:10.3390/jcm9051269

27. Napoli PE, Nioi M, Fossarello M. The "Quarantine Dry Eye": the lockdown for coronavirus disease 2019 and its implications for ocular surface health. Risk Manag Healthc Policy. 2021;14:1629-1636. doi:10.2147/RMHP.S277067

28. Ting DSJ, Krause S, Said DG, Dua HS. Psychosocial impact of COVID-19 pandemic lockdown on people living with eye diseases in the UK. Eye. 2021;35(7):2064-2066. doi:10.1038/s41433-020-01130-4

29. Konopińska J, Obuchowska I, Lisowski Ł, Dub N, Kozera M, Rękas M. Intention to get COVID-19 vaccinations among ophthalmology residents in Poland: a cross-sectional survey. Vaccines. 2021;9(4);371. doi:10.3390/vaccines9040371

30. Mathieu E, Ritchie H, Ortiz-Ospina E, et al. A global database of COVID-19 vaccinations. Nat Hum Behav. 2021:1-7. doi:10.1038/ s41562-021-01122-8

31. Napoli PE, Mangoni L, Gentile P, Braghiroli M, Fossarello M. A panel of broad-spectrum antivirals in topical ophthalmic medications from the drug repurposing approach during and after the coronavirus disease 2019 era. J Clin Med. 2020;9(8):2441. doi:10.3390/jcm9082441

32. Rezar S, Sacu S, Blum R, Eibenberger K, Schmidt-Erfurth U, Georgopoulos M. Macula-on versus macula-off pseudophakic rhegmatogenous retinal detachment following primary 23-gauge vitrectomy plus endotamponade. Curr Eye Res. 2016;41(4):543-550. doi:10.3109/02713683.2015.1031351 
33. Kim JD, Pham HH, Lai MM, Josephson JW, Minarcik JR, Von Fricken M. Effect of symptom duration on outcomes following vitrectomy repair of primary macula-off retinal detachments. Retina. 2013;33(9):1931-1937. doi:10.1097/IAE.0b013e3182877a27

34. Jasani KM, Ivanova T, Sabatino F, et al. Changing clinical patterns of rhegmatogeneous retinal detachments during the COVID19 pandemic lockdown in the North West of the UK. Eur J Ophthalmol. 2020:1120672120965480. doi:10.1177/1120672120965480.

35. Van de Put MAJ, Hooymans JMM, Los LI, Group DRRDS. The incidence of rhegmatogenous retinal detachment in the Netherlands. Ophthalmology. 2013;120(3):616-622. doi:10.1016/j.ophtha.201 2.09.001
36. Howie AR, Darian-Smith E, Allen PL, Vote BJ. Whole population incidences of patients presenting with rhegmatogenous retinal detachments within Tasmania, Australia. Clin Exp Ophthalmol. 2016;44 (2):144-146. doi:10.1111/ceo.12640

37. Rozon JP, Hébert M, Bourgault S, et al. Fear associated with COVID-19 in patients with neovascular age-related macular degeneration. Clin Ophthalmol. 2021;15:1153-1161. doi:10.2147/ OPTH.S300239

\section{Publish your work in this journal}

Clinical Ophthalmology is an international, peer-reviewed journal covering all subspecialties within ophthalmology. Key topics include: Optometry; Visual science; Pharmacology and drug therapy in eye diseases; Basic Sciences; Primary and Secondary eye care; Patient Safety and Quality of Care Improvements. This journal is indexed on PubMed

Submit your manuscript here: https://www.dovepress.com/clinical-ophthalmology-journal
Central and CAS, and is the official journal of The Society of Clinical Ophthalmology (SCO). The manuscript management system is completely online and includes a very quick and fair peer-review system, which is all easy to use. Visit http://www.dovepress.com/ testimonials.php to read real quotes from published authors. 